\title{
Reconstructing bottom water temperatures from measurements of temperature and thermal diffusivity in marine sediments
}

\author{
F. Miesner ${ }^{1,2}$, A. Lechleiter $^{1}$, and C. Müller ${ }^{2}$ \\ ${ }^{1}$ Zentrum für Technomathematik, University of Bremen, Bremen, Germany \\ ${ }^{2}$ FIELAX Gesellschaft für wissenschaftliche Datenverarbeitung mbH, Bremerhaven, Germany \\ Correspondence to: F. Miesner (fmiesner@math.uni-bremen.de)
}

Received: 20 August 2014 - Published in Ocean Sci. Discuss.: 24 October 2014

Revised: 19 June 2015 - Accepted: 24 June 2015 - Published: 09 July 2015

\begin{abstract}
Continuous monitoring of oceanic bottom water temperatures is a complicated task, even in relatively easy-toaccess basins like the North or Baltic seas. Here, a method to determine annual bottom water temperature variations from inverse modeling of instantaneous measurements of temperatures and sediment thermal properties is presented. This concept is similar to climate reconstructions over several thousand years from deep borehole data. However, in contrast, the presented method aims at reconstructing the recent temperature history of the last year from sediment thermal properties and temperatures from only a few meters depth. For solving the heat equation, a commonly used forward model is introduced and analyzed: knowing the bottom water temperature variations for the preceding years and the thermal properties of the sediments, the forward model determines the sediment temperature field. The bottom water temperature variation is modeled as an annual cosine defined by the mean temperature, the amplitude and a phase shift. As the forward model operator is non-linear but low-dimensional, common inversion schemes such as the Newton algorithm can be utilized. The algorithms are tested for artificial data with different noise levels and for two measured data sets: from the North Sea and from the Davis Strait. Both algorithms used show stable and satisfying results with reconstruction errors in the same magnitude as the initial data error. In particular, the artificial data sets are reproduced with accuracy within the bounds of the artificial noise level. Furthermore, the results for the measured North Sea data show small variances and resemble the bottom water temperature variations recorded from a nearby monitoring site with relative errors smaller than $1 \%$ in all parameters.
\end{abstract}

\section{Introduction}

The depth-dependent temperature in marine sediments is controlled by the amount of heat exchange with the water above and the deeper regions of Earth's mantle, as well as on the thermal properties of the sediment. When the water temperature is time-independent and there are no heat sinks and sources, a steady state is achieved where the vertical heat flow is constant - at least in timescales of decades.

Periodically changing water temperatures are measurable to different depths, depending on the amplitude, period and the sediment thermal properties. A reliable forward model to describe the sediment temperature in the steady state or with (periodically) changing water temperatures exists (see e.g., Lowrie, 2007) and will be introduced in Sect. 2.

Measured and modeled subsurface temperatures are analyzed for different purposes (Chouinard et al., 2007). In climate research borehole temperature data are inverted for the ground surface history (Shen and Beck, 1991). Here, the surface temperature is modeled as a Fourier series and often more than one temperature-depth profile is used. The aim is to obtain mean temperatures over time intervals of several years (Chouinard et al., 2007) and hence the mathematical models are linear but high-dimensional. The involved inversion schemes are adapted to these models. A more simple model is introduced later, where these inversion schemes are not strictly necessary.

Other studies focus on the background heat flow (e.g., Wang and Beck, 1987; Hamamoto et al., 2005). Under the circumstances where the surface temperature can be regarded as constant and no heat sinks or sources are present, the measured subsurface temperatures show a nearly linear increase 
with depth. Taking changing thermal properties of the sediment into account, the geothermal gradient can be determined directly from temperature measurements (Beardsmore and Cull, 2001). To decide from these data if the steady state geothermal gradient is prevailing, the Bullard method is applied (Bullard, 1939). If the geothermal gradient is the purpose of the investigation, a steady state is strictly required and any disturbance originating from surface temperature variations is regarded as noise. In the deep sea this is usually not an issue, as the water column already filters the surface temperature variations and the bottom water temperature is (almost) constant (Davis et al., 2003). In regions with shallower water or onshore this is a rather big problem and is often only solvable by using temperature measurements from deep boreholes.

In this work the bottom water temperature history is of interest, but on smaller timescales. The aim is to estimate the bottom water temperature variations over the last year based only on one single measured profile of depth-dependent temperature and thermal diffusivity. A parameterized function for the bottom water temperature is introduced, which results in a non-linear but low-dimensional operator. As only one measured profile is required, this could help to understand the (temperature) dynamics of water basins where continuous temperature monitoring is difficult to realize (e.g., in the Arctic Ocean).

Besides artificial data sets, results for two measured data sets from the German North Sea and the Davis Strait are presented. The regions where these measurements were performed are quite different and thus show the broad field of usability of the presented method.

\section{Forward model setup}

For the theoretical framework, the sediment is considered as a horizontally layered half space, where no temperature change happens in each of the horizontal directions and thus the one-dimensional heat equation can be applied:

$\rho C_{v} \partial_{t} T(x, t)=\partial_{x}\left(\lambda \partial_{x} T(x, t)\right)+\partial_{x}(\mathrm{v} T(x, t))+H(x, t)$,

where $x \in \Omega \subset \mathbb{R}_{\geq 0}$ corresponds to the depth below the sea floor at $x=0, t>0$ is the time, $v$ is the vertical movement of material and $H$ an inner source. The sediment density $\rho$, specific heat capacity $C_{v}$ and thermal conductivity $\lambda$ are typically depth-dependent. In the example areas, the volumetric heat capacity $\rho C_{v}$ shows only very small changes with depth and will therefore be considered as constant (see also Clauser, 2006). With the relation $\kappa=\frac{\lambda}{\rho C_{v}}$ for the thermal diffusivity the parameters can be reduced to only one per layer.

The equation above states that the deviation of the temperature $T(x, t)$ with respect to time equals the amount of diffusion (first term on the right hand side of the equation) plus the heat transported with the material by $\boldsymbol{v}$ (second term) and the generated heat $H(x, t)$. In the presented settings, the advection term $\partial_{x}(\boldsymbol{v} T(x, t))$ is neglected. In regions affected by hydrothermal convection this term can make a big difference, so data sets where the fluid flow is rather low and thus advection has no influence have been picked.

Earth's heat source is modeled as a steady state heat flow that contributes to the model via the lower boundary and thus there is no source term. With these reductions the heat equation can be simplified to the model equation:

$\partial_{t} T(x, t)-\partial_{x}\left(\kappa(x) \partial_{x} T(x, t)\right)=0 \quad \forall x>0, t>0$.

\subsection{Thermal properties and boundary conditions}

The geothermal gradient is the first derivative of the steady state solution of the model equation with a constant homogeneous boundary value at $x=0$. The steady state heat flow will be denoted with $q$ and thus $\partial_{x} T_{\text {steady }}(x, t)=\frac{q}{\lambda}=c_{\mathrm{g}}$ holds for all $x>0, t>0$.

While the bottom water temperature is constant in the steady state, in general it is time-dependent. This deviation of the bottom water temperature will be denoted by $T_{\text {water }}^{f}: \mathbb{R}_{\geq 0} \rightarrow \mathbb{R}$, where $f$ is a vector of parameters. The parameters in $f$ are to be reconstructed.

With initial and boundary conditions, the model Eq. (1) becomes a solvable initial-boundary-value problem. In geophysical models aiming for temperature fields in the earth, it is quite common to model the region $\Omega$ with infinite depth (Jaupart and Mareschal, 2011).This approach is also used here, but in the numerical implementation the region will always have a finite depth $x_{E}$ which is sufficiently large.

The boundary conditions need to be set up to satisfy the physical conditions, which are the stimulation from a 1year periodic function of the bottom water temperature and a zero-flow condition at the lower boundary. The geothermal gradient as the solution to the static heat equation will be added after the modeling process. Thus, a homogeneous Neumann condition can be set at the lower boundary in the time-dependent part and the Robin boundary condition at the sediment-water interface. The latter describes the fact that the heat flows out of the sediment when the sediment is warmer than the water above and into the sediment when it is cooler. 
Knowing the thermal diffusivity $\kappa(x)$ of the sediment and the parameters $f \in \mathcal{D}_{T}$ of a continuous function $T_{\text {water }}^{f}(t)$ for the bottom water temperature, this yields a full set of equations to determine the temperature in marine sediment at every place and time: when $u(x, t)$ solves Eqs. (2)-(5), the sediment's temperature is given by $T_{\text {total }}(x, t)=u(x, t)+c_{\mathrm{g}} x$,

$$
\begin{array}{lr}
\partial_{t} u(x, t)-\partial_{x}\left(\kappa(x) \partial_{x} u(x, t)\right)=0 & \forall x \in \Omega, t>0, \\
\kappa(x) \partial_{x} u(0, t)=\frac{h}{\rho c_{v}} \cdot\left(u(0, t)-T_{\text {water }}^{f}(t)\right) & \forall t>0, \\
\partial_{x} u\left(x_{E}, t\right)=0 & \forall t>0 \text { if } x_{E}<\infty, \\
\lim _{x \rightarrow \infty} \partial_{x} u(x, t)=0 & \forall t>0 \text { if } x_{E}=\infty, \\
u(x, 0)=u_{0}(x) & \forall x \in \Omega .
\end{array}
$$

Here, $h(t)$ is the heat transfer coefficient and a measure on how well the heat energy passes the sediment-water interface. Brakelmann and Stammen (2006) discuss the value of this coefficient and propose to use an average value of $h=150 \mathrm{~W} \mathrm{~m}^{-2} \mathrm{~K}$ for the German North and Baltic seas. They also showed that the influence of deviations of this parameter is negligible and therefore this constant value will be used throughout the paper. The volumetric heat capacity $\rho c_{v}$ is also assumed to be known from measurements.

\subsection{Bottom water temperature functions}

The temperature at Earth's surface is an overlay of many sinusoidal functions with different periods in terms of Fourier series. As the deviation of the bottom water temperature for 1 year is the aim of the reconstruction, the following simple model with only a 1 -year period $\omega=\frac{2 \pi}{365}$ is used:

$T_{\text {water }}^{f}(t)=A+B \cos (\omega t+\varphi(d)), \quad f=(A, B, d)^{T}$,

where $A$ and $B$ (in ${ }^{\circ} \mathrm{C}$ ) denote the average temperature and amplitude, respectively. The annual minimum takes place on a day $d>0$, which leads to a phase delay $\varphi(d)=\omega\left(\frac{365}{2}-\right.$ $d$ ) of the cosine function, where the definition space of $f$ is restricted to $\mathcal{D}_{T}=\mathbb{R}_{\geq 0} \times \mathbb{R}_{\geq 0} \times[0,365]$.

The example data set from the German North Sea shows influences of smaller periods besides the annual deviation, as the average depth is only $100 \mathrm{~m}$ (Rhode et al., 2004). Temperature and salinity of the North Sea are mainly governed by a general cyclonic circulation, which renews the water in the timescale of 1 year (Rhode et al., 2004). The freshwater input from rivers is comparably small. The central part of the North Sea becomes stratified due to heating in the summer but gets vertically mixed during winter. At the western and southern coasts, the vertical stratification is prevented by strong tidal currents (for detail on the oceanography of the German North Sea see Rhode et al., 2004). Thus, in the North Sea the simple model for the bottom water temperature is a sufficient approximation. However, the temperature deviation will be slightly noisy due to the shallow depths. Also, the parame- ters will change slightly in different regions, relating to the tidal currents.

Baffin Bay and Davis Strait are characterized by the northwards flowing West Greenland Current moving temperate saline Irminger Water from the Atlantic Ocean in the top layers and cold low-salinity Polar Water in the bottom layers (Ribergaard, 2008). The data set from the Davis Strait is obtained from $1300 \mathrm{~m}$ depth, where the seasonal influence is mostly damped by the water column and the cold Polar Water is dominant. Thus, the parameters $A$ and specifically $B$ are expected to be near $0^{\circ} \mathrm{C}$ (Ribergaard, 2008).

\subsection{General behavior}

The general behavior of the forward model is depicted in Fig. 1. A parameter set typical for the German North Sea of $f=(10.5,7,45)^{T}$ is used for the bottom water deviation. For the geothermal gradient a literature value of $c_{\mathrm{g}}=$ $0.03 \mathrm{Km}^{-1}$ (Global HF DB, 2014) is used. The image shows that the sediment experiences a large temperature range over 1 year in the upper meters. As the depth increases, the covered temperature range gets smaller.

The attenuation of the amplitude with depth is clearly visible and so is the delay of the temperature. It can be observed that the current temperature-depth profile contains the bottom water temperatures from the last 3-4 months. Following the orange line indicating the day of the highest bottom water temperature, the sediment temperature shows a decrease with depth. At a depth of $4 \mathrm{~m}$ the lowest temperature, as evidence of the last winter, is reached and with further increasing depth the temperatures also increase again. The blue line indicating the day of the coldest bottom water temperature shows a mirror-inverted curve.

\section{Data}

The temperature reconstructing method (to be described in Sect. 4) was tested for artificial and measured data sets containing about 20 data points in a depth of up to $4 \mathrm{~m}$. The artificial data sets were generated using the forward model and adding some white noise, while the example data sets from the German North Sea and the Davis Strait were measured using the FIELAX VibroHeat and HeatFlow measuring devices, respectively. The locations of the example data sets are shown in Fig. 2.

The principle for the measurements of depth-dependent thermal parameters originates from the classical method of determining steady state heat flow values for the oceanic crust from deep sea sediments. Heat flow values are determined based on Fourier's law of thermal conduction from the steady state (undisturbed) temperature gradient and thermal conductivities. The design of deep sea Lister-type heat flow probes follows the concept by Hyndman et al. (1979) where a thermistor string parallel to a massive strength mem- 

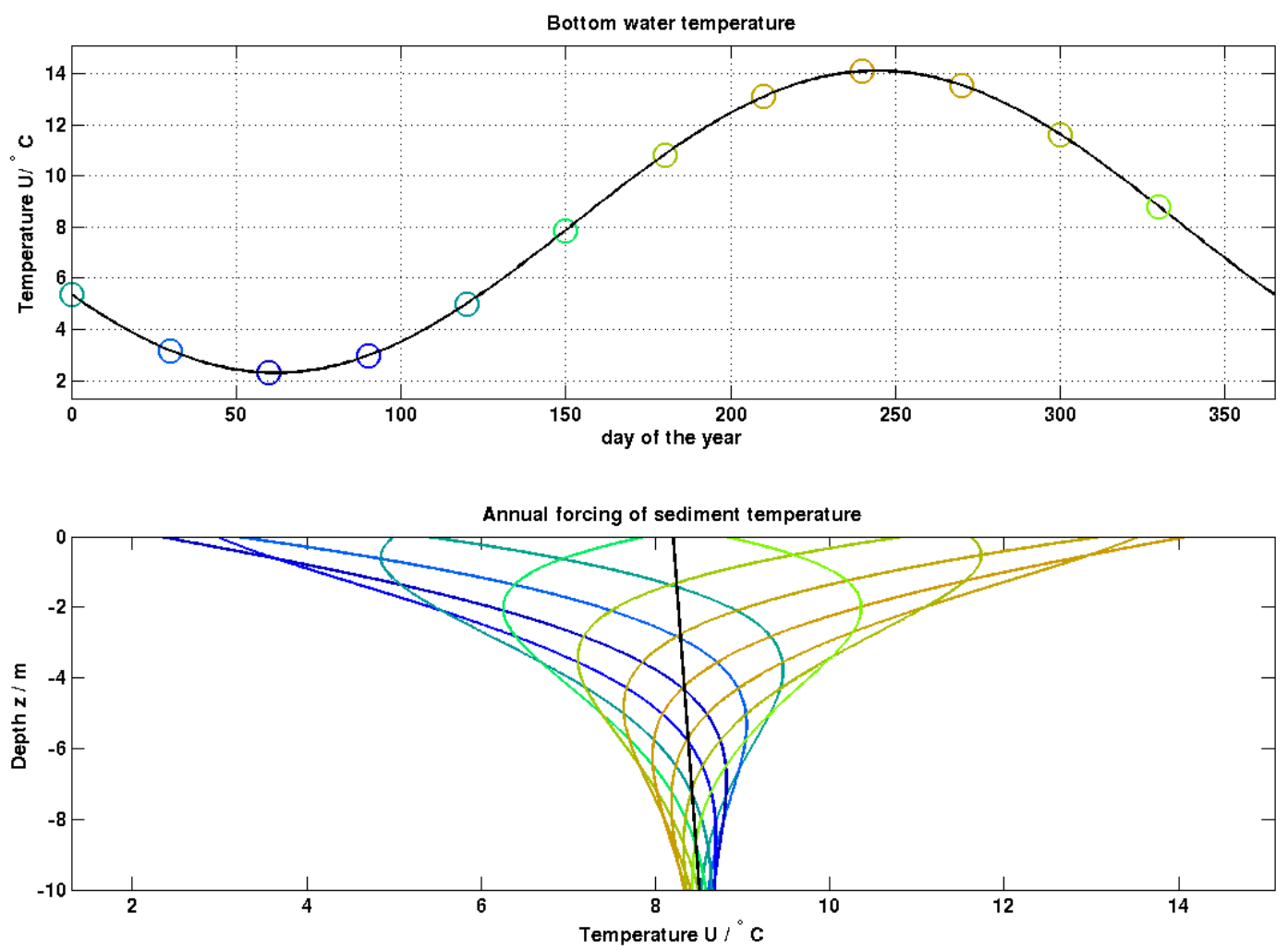

Figure 1. Bottom water temperature (top panel) as input to the forward model operator and the solution (bottom panel) at different days of the year. A constant thermal diffusivity of $\kappa=8 \times 10^{-7} \mathrm{~m}^{2} \mathrm{~s}^{-1}$ was used and the geothermal gradient (black in the lower panel) was set to $c_{\mathrm{g}}=0.03 \mathrm{Km}^{-1}$. The bottom water temperature function is a cosine with a mean value of $8.2^{\circ} \mathrm{C}$, an amplitude of $\pm 5.9 \mathrm{~K}$ and the minimum on the 62 day of the year. The colors indicate the bottom water temperature and thus the day of the year when the temperature-depth profile is plotted. The bright orange temperature-depth profile is the solution on the 220th day of the year, when the bottom water temperature is near the annual maximum of $14.1^{\circ} \mathrm{C}$.

ber penetrates into the sediment by its own weight. A total of 22 thermistors record the sediment temperature during the whole process. The in situ temperature is determined from the decay of the frictional heat accompanying the penetration, while the thermal conductivity and diffusivity values are calculated from the temperature decay of an artificial, exactly defined, calibrated heat pulse which heats up the sediment (Hartman and Villinger, 2002).

This method is normally used in deep sea soft sediments, where the heat flow probe penetrates due to its own weight. Shallow water sediments in the North and Baltic seas are characterized by more shear resistant sediments such as sands, tills, and clays, where this classical method of penetration by gravity alone is not applicable. For this reason, a thermistor string has been combined with a standard VKG vibrocorer (Dillon et al., 2012). The measuring procedure follows the classical way: the system is lowered towards the sediment, penetrates the sediment by vibrocoring and rests in the sediment for in situ temperature measurement and heat pulse decay recording. With this system, a penetration depth of up to $6 \mathrm{~m}$ is possible. The accuracy of the thermistor strings is $2 \mathrm{mK}$ and the resolution is $1 \mathrm{mK}$ for both systems.
The processing of the raw data from both measuring devices is handled with the same software tool. This processing algorithm allows determining in situ temperatures and thermal material properties with an inversion algorithm following Hartman and Villinger (2002). Based on an assumption of thermal decay around a cylindrical symmetric infinite line source, steady state in situ temperatures, thermal conductivity and thermal diffusivity are determined in an iterative inversion procedure. From thermal conductivity and diffusivity also the volumetric thermal capacity can be determined by $\rho c_{v}=\lambda / \kappa$. All thermal properties can be obtained with less than $0.5 \%$ error.

The in situ geothermal gradient can also be determined directly from these measurements (for details see Beardsmore and Cull, 2001). When the measurements are not deep enough, the determination of the in situ geothermal gradient is not possible. The global heat flow database collects data sets and can thus be used to find an appropriate regional approximation (Global HF DB, 2014). The proposed inversion method is based on routine thermal measurements aiming for the geothermal heat flow. In the processing of these measurements with the algorithm from Hartman and Villinger 

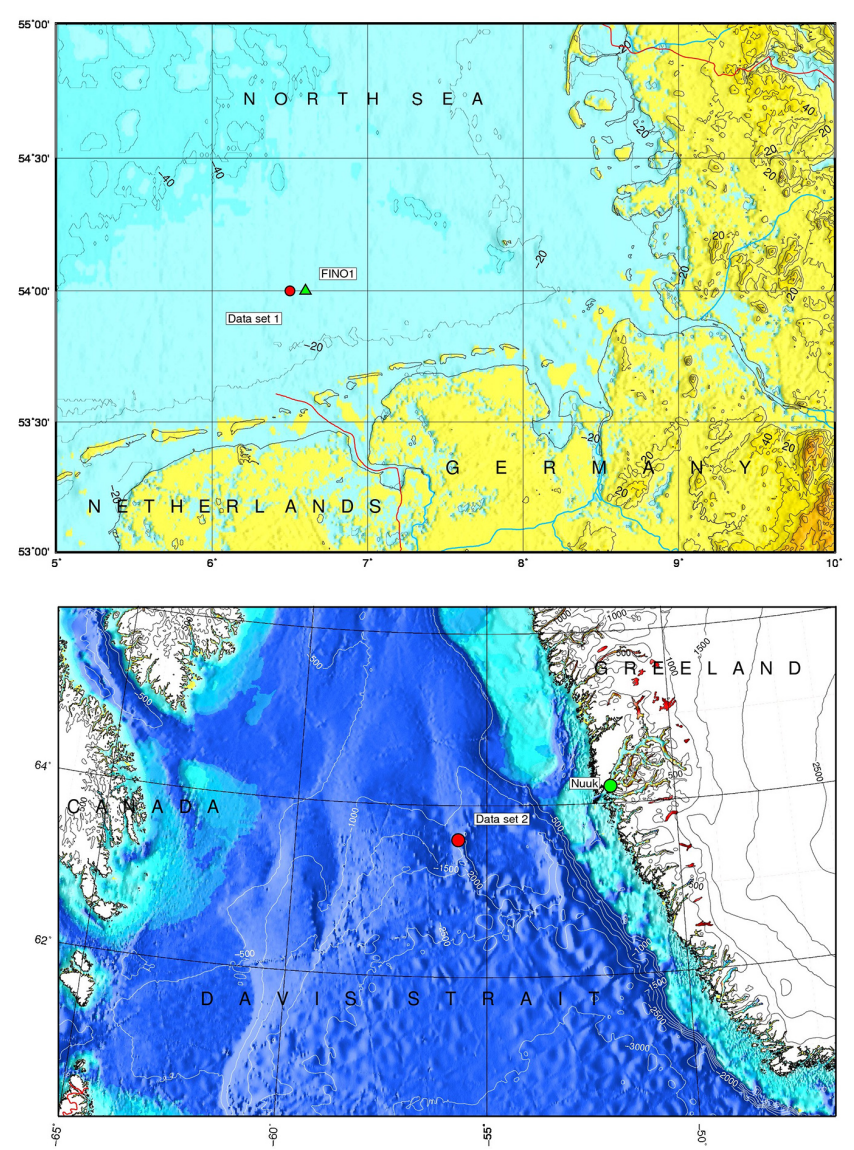

Figure 2. Locations of the two example data sets (red). In the upper panel, the data's location near the island of Borkum in the German North Sea is depicted. Additionally the observation station FINO1 is marked (green). In the lower panel, the data's location west of the coast of Greenland, near Nuuk, is shown.

(2002), the in situ thermal properties and the geothermal heat flow are determined. Any sedimentation effects are dealt with in this algorithm and can thus be omitted in the inversion schemes. Therefore, the geothermal heat flow is a known input to the model, and approximated values are used where the in situ determination was not possible. A simultaneous inversion of the bottom water function parameters together with the heat flow could decrease the influence of measurement errors; however, this will not be handled in this paper.

Figure 3 shows depth-dependent properties from a thermal measurement in the North Sea offshore from Borkum in June 2011. Contrary to the general positive temperature gradient in Earth's crust, a temperature decrease with increasing depth is observed, i.e., a negative temperature gradient. This is due to the seasonal variation of bottom water temperature in the North Sea. As the measurement was performed in June, the influence of the warm summer temperatures is seen in the upper thermistors, while the decrease towards the lower thermistors is a relict of low winter temperatures.
The inversion scheme was performed for various data sets from the German North and Baltic seas being measured with the VibroHeat device and data sets from the Davis Strait and the Baffin Bay, which were measured using the classic HeatFlow probe. Excluding some measurements from areas within the Baltic Sea where the bottom water temperature deviation differs too much from the simple model Eq. (6), the obtained results were all within the same range of quality and accuracy. The two data sets presented here are chosen for demonstration of the method.

\section{Inverse problem setup}

\subsection{Discretization of the forward model operator}

The inverse problem is to determine the bottom water parameters $f$ from (a priori known) values for given geothermal gradient $c_{\mathrm{g}}$, heat transfer coefficient $h$ for the Robin boundary condition, and measurements of the thermal diffusivity $\kappa(\widetilde{x})$ and the temperature in the sediment $g^{\varepsilon}\left(\widetilde{x}, t^{*}\right)$. Here, $\tilde{x} \in \mathbb{R}^{k}$ is the depth vector according to the $k$ sensors of the measuring device and $t^{*}>0$ a fixed time.

Before introducing the solution method to solve this inverse problem, the forward model needs to be briefly formalized. It can be shown that the initial-boundary problem, Eqs. (2)-(5), has a unique solution in the weak sense (see e.g., Evans, 2010). Thus, the forward operator $F: f \longmapsto$ $T(x, t)$ mapping the parameters of the bottom water temperature to the solution of the initial-boundary problem is well-defined. For this operator, differentiability with respect to $f$ can be shown if the function for the bottom water temperature deviation $T_{\text {water }}^{f}$ is continuously differentiable with respect to the parameters $f$. The continuous differentiability of the cosine function in Eq. (6) with respect to the three parameters $A, B$ and $d$ is obvious.

When interested in greater timescales it is common to model Earth's crust as a homogeneous half space, i.e., $\Omega$ has infinite depth and the thermal diffusivity $\kappa$ is constant over the region. In this case, the solution to the initial-boundary problem is analytical. Thus, in the numerical calculation of the temperature in the sediment the only occurring errors are rounding errors on the scale of the machine accuracy and no discretization errors. However, as the sediment is modeled as a layered region with finite depth there are two more sources of error: the discretization error and an error resulting from the finite depth.

The discretization is realized using the method of lines. This method and its convergence properties are broadly analyzed by Hanke-Bourgeois (2009). The mesh size and time steps of the discretization as well as the depth of the lower boundary need to be determined such that the numerical solution is not too far away from the true solution. In other words, the scheme determines a parameter setting such that the relative error between the abovementioned analytical so- 

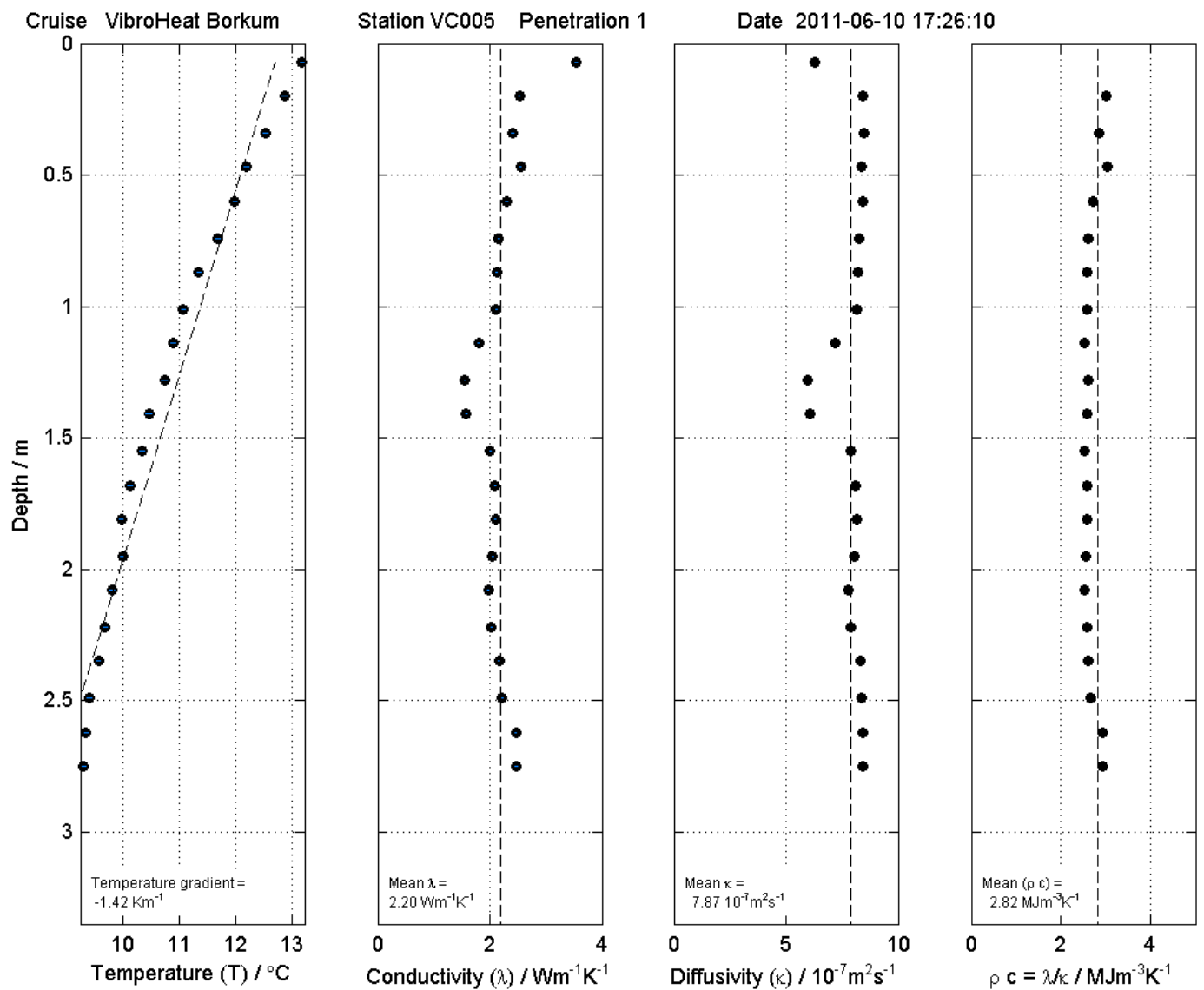

Figure 3. Results of a VibroHeat survey in the North Sea north of Borkum in 2011 showing in situ temperature, thermal conductivities, thermal diffusivity, and volumetric heat capacity as a function of depth.

lution and the numerical solution does not exceed the limit of $10^{-3} \mathrm{~K}$. This limit was chosen in reference to the accuracy of the data obtained with the FIELAX VibroHeat device.

Having access to a numerical and nonlinear forward model $F: \mathbb{R}^{3} \rightarrow \mathbb{R}^{k}$ mapping the three model parameters $A, B$ and $d$ to the numerically approximated temperature for time $t_{*}>$ 0 at points of the depth vector $\tilde{x} \in \mathbb{R}^{k}$, the inverse method can be discussed based on the observation that $F$ possesses a gradient $\nabla F \in \mathbb{R}_{k \times 3}$. This matrix had maximal rank (3) in all performed numerical examples.

Since the measured sediment temperature (and also the measured thermal diffusivity) may suffer from measurement errors, the temperature data are assumed to be a vector $g^{\varepsilon}$ such that $\left\|g-g^{\varepsilon}\right\| \leq \varepsilon$, where $g$ is the undisturbed data. Furthermore, an exact parameter vector $f^{+}$is assumed to exist such that $F\left(f^{+}\right)=g$ (i.e., the model is valid and represents reality). The aim of the inversion scheme is to reconstruct $f^{+}$.

The study started with a non-linear iterative Newton algorithm as a first simple approach, which already provided good results. Therefore, this approach is discussed first. For comparison the iterative REGINN (REGularization by INex- act Newton methods; Rieder, 2003) method has been adapted to the model.

\subsection{The Newton algorithm}

Sticking to the notation of Rieder (2003), the ongoing iteration for the solution of the non-linear equation $F(f)=g^{\varepsilon}$ with disturbed data $g^{\varepsilon}$ is considered:

$f_{n+1}^{\varepsilon}=f_{n}^{\varepsilon}+s_{n}^{\varepsilon}, \quad n \in \mathbb{N}$.

The iteration step $s_{n}^{\varepsilon}$ is to be determined, so that the exact solution $f_{n+1}^{\varepsilon}=f^{+}$is obtained. Obviously, $s_{n}^{+}=f^{+}-f_{n}^{\varepsilon}$ solves this equation. The approach of the Newton algorithm is to determine a good approximation to $s_{n}^{+}$.

As $F$ is differentiable with derivative $\nabla F$, the following equation holds:

$\nabla F\left(f_{n}^{\varepsilon}\right) s_{n}^{+}=g-F\left(f_{n}^{\varepsilon}\right)-E\left(f^{+}, f_{n}^{\varepsilon}\right)=b_{n}$.

Here, $E(v, w)$ denotes the linearization error. This linearization error and therefore the right side $b_{n}$ is not known but only a disturbed version $b_{n}^{\delta}$ is. So $s_{n}^{+}$is approximated by solving

$\nabla F\left(f_{n}^{\varepsilon}\right) s=b_{n}^{\delta}$. 
For ill-posed problems, solving this linear equation can be quite problematic; however, as the derivative has full rank, Gaussian elimination can be used to determine $s_{n}^{\varepsilon}$.

Applied to the simple model, the iteration converges to a solution of $F(f)=g^{\varepsilon}$. However, as the method is not minimizing for the exact right side $b_{n}=g-F\left(f^{+}\right)$but for a disturbed version, the best result may not be the minimal result. Therefore, the iteration is stopped whenever the reconstructed data are about as near to the noisy data $g^{\varepsilon}$ as the noisy data are away from the exact data. Details on this discrepancy principle can be found, e.g., in Rieder (2003). As the value of $\varepsilon$ is not known for real data sets, an approximation of $\varepsilon=0.005$ is used.

\subsection{A regularized inexact Gauss-Newton inverse method}

For the inversion of the simple model, Eq. (6), for the bottom water temperature deviation, the Newton algorithm yielded stable results (as will be shown in Sect. 5), such that stabilizing the inversion by using a so-called regularization scheme was not necessary. Such schemes can balance stability and accuracy of a solution to an inverse problem (see Rieder, 2003) and could be of importance if a more complex input model for the bottom water temperature is used. This will not be covered in this work, but will be discussed as a suggestion for further research in the last section. One advantage of regularization schemes in general and of the REGINN algorithm published by Rieder (2003) is their proven convergence properties for noisy data or ill-conditioned non-linear equations.

The REGINN algorithm is introduced and analyzed by Rieder (1999a) and Rieder (1999b). The algorithm is an inexact Newton method, i.e., it consists of an outer Newton iteration and an inner regularization iteration which determines the Newton iteration step. Thus, the outer iteration follows the same idea as the abovementioned simple Newton method, Eq. (7), where the iteration step $s_{n}^{\varepsilon}$ solves Eq. (8), and which is stopped with the discrepancy principle. The determination of the iteration steps can be implemented with any regularization scheme and the analysis is done for a general formulation.

For this work, the algorithm published by Rieder (2003), where the inner iteration is a conjugate gradient (CG) method, was adapted. As the algorithm has two nested iterations, the outer (Newton) iteration will be referred to with superscript $n$ and the inner (CG) iteration with superscript $m$.

The idea of the algorithm starts again with Eq. (8),

$\nabla F\left(f_{n}^{\varepsilon}\right) s=b_{n}^{\delta}$,

for the Newton iteration step $s_{n}^{\varepsilon}$ and tackles this linear system using the $\mathrm{CG}$ method.

The CG method is designed to minimize the residuum $\left\|b_{n}^{\delta}-\nabla F\left(f_{n}^{\varepsilon}\right) s_{m}\right\|$ in every iteration step $m=1,2,3, \ldots$ in enlarging subspaces. As the residuum at the end of every iter- ation step is the minimum in the corresponding subspace, the method is the most efficient method possible. For more information on the CG method see e.g., Rieder (2003) and HankeBourgeois (2009). The inner iteration is stopped when the linearized residuum fulfills a certain accuracy estimation (for details on the choice of this estimation see Rieder, 2003). The outer iteration is again stopped with the discrepancy principle. Thus, the reconstructions from the two algorithms can be directly compared.

\section{Results}

\subsection{Artificial data for measured thermal diffusivity}

In this section, the sensitivity of the algorithms in a layered half-space setting is analyzed. Therefore, the measured thermal diffusivity and the depth-vector of the sensors are used together with a random day of the year $t_{*}$ and a random vector of the seasonal forcing parameters to produce artificial data. With added zero-mean Gaussian noise, disturbed data sets $g^{\varepsilon}$ were obtained. For comparability of the results, the same water parameters $f_{\text {Exact }}=(8.2,5.9,62)^{T}$ and literature values for $c_{\mathrm{g}}$ and $h$ are used for all the experiments presented here.

As the derivative is not a square matrix, any theoretical analysis of the convergence behavior of the Newton or REGINN algorithm does not apply. To cope with this lack of theoretical information on the general behavior of the introduced inverse problem, the algorithms were executed repeatedly for randomly disturbed artificial data sets, produced from the same parameters, and the variances were calculated. Thus, a bound for the emerging reconstruction error depending on the noise level in the data can be given. The results are shown in Table 1.

For data with a noise level of $0.1 \%$ (see Table 1 ) the reconstruction error has the same magnitude for both algorithms. As the initial guess is chosen randomly from the definition space and the variances are very small, it can be stated that both algorithms work stably and that the initial guess has no influence.

For a noise level of $1.0 \%$ in the data, a maximum reconstruction error of about $8 \%$ is obtained, while the mean value of all reconstructions is still close to the exact parameter values with a mean error of only $3 \%$ (see Table 1) with both algorithms. This suggests that the overall result of the inversion can be improved by executing the algorithm several times for different (randomly chosen) intial guess every time and using the resulting mean value.

The overall mean parameter values $f_{\text {overall }}=$ $(8.29,6.11,62.6)^{T}$ yield a relative reconstruction error of $\approx 1.1 \%$. 
Table 1. Statistics for the inversion of artificial data. The uppermost part contains the inversion results for artificial data with $0.1 \%$ white noise added, the middle part artificial data with $1.0 \%$ noise. The lowermost part shows inversion results to artificial data with $0.1 \%$ white noise added after the seasonal forcing already contained noise of about $8 \%$.

\begin{tabular}{|c|c|c|c|c|c|}
\hline Data & \multicolumn{3}{|c|}{ Artificial with $0.1 \%$ noise } & & \\
\hline \multirow[t]{2}{*}{ exact parameters } & $A$ & $B$ & $d$ & & \\
\hline & 8.2 & 5.9 & 62 & & \\
\hline Newton results & $A$ & $B$ & $d$ & mean error & $\max$ error \\
\hline \multirow{2}{*}{$\begin{array}{l}\text { mean } \\
\text { variances }\end{array}$} & 8.20 & 5.89 & 61.97 & $0.31 \%$ & $0.65 \%$ \\
\hline & 0.00 & 0.00 & 0.05 & & \\
\hline \multirow{3}{*}{$\begin{array}{l}\text { REGINN result } \\
\text { mean } \\
\text { variances }\end{array}$} & $A$ & $B$ & $d$ & mean error & $\max$ error \\
\hline & 8.20 & 5.90 & 61.96 & $0.21 \%$ & $0.59 \%$ \\
\hline & 0.00 & 0.00 & 0.03 & & \\
\hline Data & \multicolumn{3}{|c|}{ Artificial with $1.0 \%$ noise } & & \\
\hline \multirow[t]{2}{*}{ exact parameters } & $A$ & $B$ & $d$ & & \\
\hline & 8.2 & 5.9 & 62 & & \\
\hline \multirow{3}{*}{$\begin{array}{l}\text { Newton results } \\
\text { mean } \\
\text { variances }\end{array}$} & $A$ & $B$ & $d$ & mean error & max error \\
\hline & 8.22 & 5.96 & 61.84 & $2.88 \%$ & $7.29 \%$ \\
\hline & 0.05 & 0.25 & 2.90 & & \\
\hline \multirow{3}{*}{$\begin{array}{l}\text { REGINN result } \\
\text { mean } \\
\text { variances }\end{array}$} & $A$ & $B$ & $d$ & mean error & max error \\
\hline & 8.36 & 6.26 & 63.45 & $2.63 \%$ & $5.68 \%$ \\
\hline & 0.02 & 0.13 & 1.64 & & \\
\hline Data & \multicolumn{5}{|c|}{ Artificial with $1.0 \%$ noise and $8.0 \%$ water noise } \\
\hline \multirow[t]{2}{*}{ exact parameters } & $A$ & $B$ & $d$ & & \\
\hline & 8.2 & 5.9 & 62 & & \\
\hline \multirow{3}{*}{$\begin{array}{l}\text { Newton results } \\
\text { mean } \\
\text { variances }\end{array}$} & $A$ & $B$ & $d$ & mean error & $\max$ error \\
\hline & 8.25 & 5.90 & 62.88 & $6.86 \%$ & $17.54 \%$ \\
\hline & 0.29 & 2.22 & 22.72 & & \\
\hline \multirow{3}{*}{$\begin{array}{l}\text { REGINN result } \\
\text { mean } \\
\text { variances }\end{array}$} & $A$ & $B$ & $d$ & mean error & max error \\
\hline & 8.29 & 5.89 & 62.27 & $8.69 \%$ & $14.92 \%$ \\
\hline & 0.99 & 10.07 & 29.51 & & \\
\hline
\end{tabular}

As the penetration depth of the first sensor is not exactly known, the algorithm was also tested for vertically shifted data. Here, the distance between two thermistors, e.g., $13.5 \mathrm{~cm}$ for the Borkum data, was added to the depth values. With, again, 20 repetitions of each algorithm, the mean values and the variances only differed from the non-shifted data in the third decimal place.

\subsection{Artificial data with noisy bottom water temperature}

In Sect. 5.1, artificial data for an undisturbed bottom water temperature function was produced. However, from the water data available via MARNET (2014) for the German North Sea it is known that this undisturbed function is very unlikely to be accurate. In this section, the influence of noise in the bottom water temperature on the accuracy of the reconstruction shall be investigated.
Different from the white noise added to the data itself, here shorter periods of the cosine series are used to approximate the occurring errors as accurately as possible. Thus, this section will give an insight into the sensitivity of the model with respect to the three main parameters, even if the original forcing was more complicated and the measured data contains errors.

For generating artificial data in this section, the seasonal forcing was expanded to the first 52 summands of the Fourier series for 1-year periodic functions:

$$
\begin{aligned}
& T_{\text {water }}^{f}(t):=A+\sum_{i=1}^{52} B_{i} \cos \left(i \omega t+\varphi\left(d_{i}\right)\right), \\
& f=\left(A, B_{1}, \ldots, B_{52}, d_{1}, \ldots, d_{52}\right)^{T} .
\end{aligned}
$$

This cutoff Fourier series approximates periods from 1 year to 1 week. 
The results of this experiment are presented in the last section of Table 1. A distinct increase in the reconstruction error can be observed for a noise level in the data of $1 \%$ from a mean error of $3 \%$ for an undisturbed bottom water temperature function to a mean error of $7 \%$ here. Although the variances increase, the mean reconstruction result is still a very good approximation to the exact water parameters. The reconstruction error of a single reconstruction may be greater than $15 \%$, while already 20 repeated executions can decrease the reconstruction error notably. For both algorithms, the differences between the average results and the exact parameter values are smaller than $0.1 \mathrm{~K}$ in the average temperature and the amplitude and smaller than 1 day in the day of the annual minimum. The main uncertainty occurs from the determination of the day of the annual minimum.

From the inversions done here, it can be concluded that the three main parameters get reconstructed quite well even if the real bottom water temperature function is more complicated than the simple model in Eq. (6).

\subsection{Example: Borkum}

Data sets from three different locations in the German North and Baltic seas were studied, but only one example will be presented in detail. The location of the thermal measurement and the nearest MARNET station (see MARNET, 2014) are depicted in Fig. 2. Along with two other examples, this data set is broadly discussed by Müller et al. (2013). The quality of the reconstruction results in terms of variances was the same in all studied examples. The data set north of the island of Borkum was chosen for the following reasons: the distance between the data set location and the nearest MARNET station FINO1 is smaller than with the other examples and the recorded water temperatures showed the smallest differences from the chosen bottom water temperature function Eq. (6). Additionally, the time series of the water temperatures was available and allowed analyzing the applicability of this model equation. As the measurement is only $3 \mathrm{~m}$ deep, the in situ geothermal gradient could not be obtained from the measurements. Thus, a typical value of $c_{\mathrm{g}}=0.03 \mathrm{Km}^{-1}$ for this area (Global HF DB, 2014) was used.

The results of the inversion are listed in Table 2. In comparison to the parameter vector used in Müller et al. (2013), $\widetilde{f}_{\text {Borkum }}=(10.4,6.9,41)^{T}$, the overall mean parameters fit quite well. The Newton algorithm provides smaller values than the REGINN algorithm but also with smaller variances in the average temperature and amplitude. The day of the annual minimum resulting from the Newton algorithm seems very unlikely and also has a larger variance.

Averaging all reconstructions with both algorithms, the values fit to the educated guess. The variance on the day of the annual minimum is here quite large, because it was reconstructed differently by the two algorithms. The value corresponds to a SD (standard deviation) of $\approx 15$ days.
Table 2. Results of the inversion of the data set from Borkum. The Newton algorithm gives an unlikely estimate for the day of the annual minimum but the overall mean parameters fit the guess from Müller et al. (2013) within the desired bounds.

\begin{tabular}{lccc}
\hline Data & Borkum & & \\
\hline Newton results & $A$ & $B$ & $d$ \\
mean & 9.79 & 5.66 & 26.62 \\
variances & 0.00 & 0.02 & 2.63 \\
\hline REGINN result & $A$ & $B$ & $d$ \\
mean & 11.95 & 9.15 & 55.53 \\
variances & 0.03 & 0.09 & 1.71 \\
\hline overall results & $A$ & $B$ & $d$ \\
mean & 10.87 & 7.40 & 41.07 \\
variances & 1.20 & 3.14 & 213.78 \\
\hline
\end{tabular}

Bottom water temperature data in this area were available from the FINO1 station. Inverting the water data with the same algorithm, the parameter vectors $f_{2010}=$ $(10.0,7.9,56)^{T}$ for the data from 2010 and $f_{2011}=$ $(10.4,7.1,55)^{T}$ for 2011 , respectively, were obtained. In this particular case, the average temperature and the amplitude differ less than $1 \mathrm{~K}$ between 2 years and also the day of the annual minimum remains nearly the same. This leads to the assumption that the simple model for the bottom water temperature fits the natural conditions in this area quite well.

Comparing these parameter vectors to the ones obtained from the inversion (Table 2), it is obvious that the Newton algorithm provided too small values, while the REGINN algorithm yielded too large values. The overall mean fits best, but the day of the annual minimum was reconstructed better with the REGINN algorithm alone.

In the upper panel of Fig. 4, the FINO1 data from 2010 and 2011 and, additionally, the cosine functions of the mean inversion results are shown. In the lower panel the measured thermal diffusivity (right) and the measured and modeled temperature-depth profiles are depicted. The temperature interval resulting from the Newton results is too small, while the REGINN result has too high temperatures in the second half of the year. From the three cosine functions, the one resulting from the overall mean fits the recorded temperatures best.

For the temperature-depth profile on the day of the measurement this does not hold. The model with the overall mean result has too high temperatures. The REGINN result fits the measured temperatures better but only to a depth of $2 \mathrm{~m}$, while the Newton results fit better below $2 \mathrm{~m}$ depth. The notso-optimal fit of the overall mean results can be due to the uncertainty of the reconstruction of the day of the annual minimum. By shifting the cosine of the overall mean results about 1 week (such that $d \approx 48$ ), the cosine fits the recorded temperatures at FINO1 better and the temperature-depth profile fits the measurements better as well. 

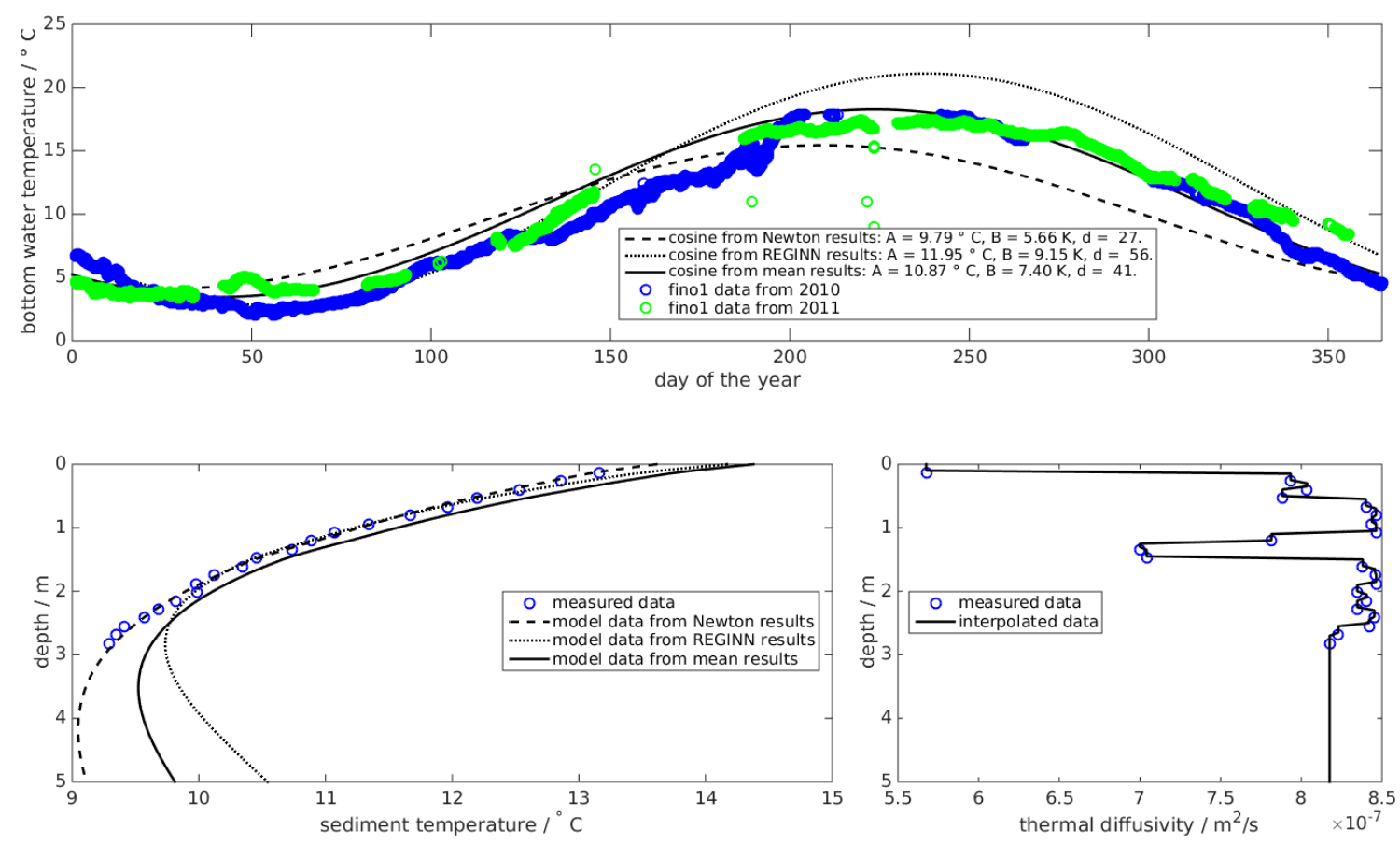

Figure 4. Inversion of the data set near Borkum. In the upper panel, the recorded bottom water temperatures at the BSH station FINO1 are depicted for the years 2010 (green) and 2011 (blue). Additionally, the cosine functions as results of the inversion schemes are plotted: the mean result of the Newton algorithm, the REGINN result, and the overall mean. In the lower panels, the measured temperature (left) and thermal diffusivity (right) are depicted. The resulting temperature-depth profiles from the modeling with the inversion results are plotted together with the measured temperatures in the left panel.

Table 3. Results of the inversion of the data set west of Greenland. Both algorithms give similar reconstruction values with small variances.

\begin{tabular}{lccc}
\hline Data & Greenland & & \\
\hline Newton results & $A$ & $B$ & $d$ \\
mean & 3.18 & 0.16 & 71.51 \\
variances & 0.00 & 0.00 & 2.15 \\
\hline REGINN result & $A$ & $B$ & $d$ \\
mean & 3.18 & 0.16 & 74.25 \\
variances & 0.00 & 0.00 & 0.93 \\
\hline overall results & $A$ & $B$ & $d$ \\
mean & 3.18 & 0.16 & 72.88 \\
variances & 0.00 & 0.00 & 3.43 \\
\hline
\end{tabular}

\subsection{Example: Greenland}

The second example data set was measured on a cruise in 2006 in the waters of the Davis Strait and Baffin Bay, west of Greenland, the location is shown in the lower panel of Fig. 2. The in situ geothermal heat flow of $c_{\mathrm{g}}=0.0303 \mathrm{Km}^{-1}$ was determined from these measurements.

The measurement is located at the southern ridge of the Davis Strait, at the passage to the Labrador Sea. The water depth is about $1300 \mathrm{~m}$ and thus the bottom water temperature deviation is expected to be rather small.

The reconstruction results are shown in Table 3. Both algorithms reconstructed similar values. As continuous measurements of the bottom water temperature deviation are not easy in these parts of the Arctic Ocean, there are no measurements to compare these values to. However, the obtained temperature interval seems plausible.

In Fig. 5, the cosine functions with the reconstructed bottom water function parameters are depicted (upper panel). In the lower panels the measured thermal diffusivity (right) and sediment temperature (left) as well as the modeled temperature are shown. The sediment shows a wide range of thermal diffusivity values. The reconstructed bottom water temperature deviations do not differ much, nor do the modeled sediment temperatures. They all fit the measured temperatures quite well. Looking at the low variances (Table 3), this indicates a stable method and high applicability.

\section{Discussion}

The aim of this work was to provide a method that obtains the parameters of a function modeling the annual bottom water temperature variation from one instantaneous measured profile of depth-dependent sediment temperature and thermal 

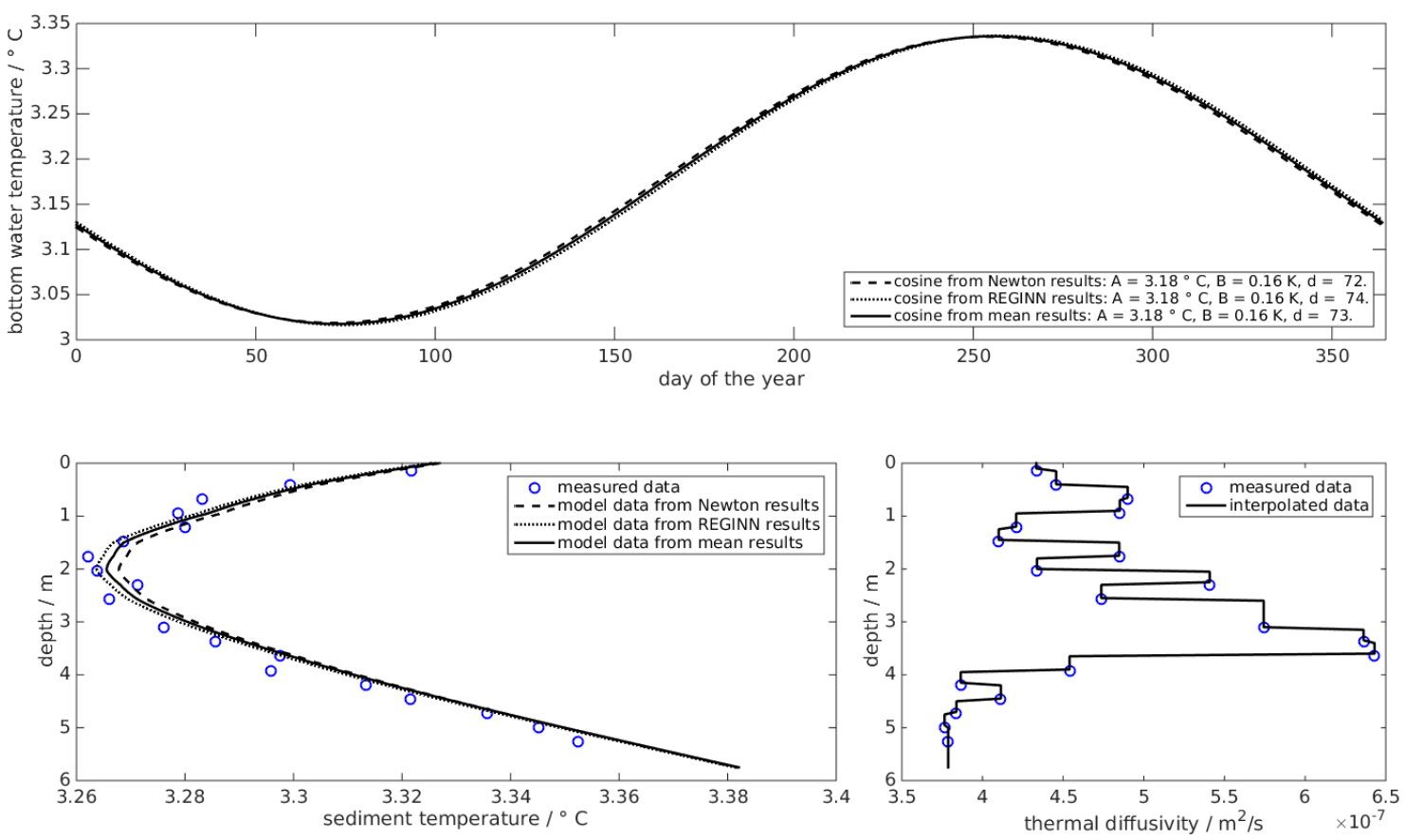

Figure 5. Inversion of the data set west of Nuuk. In the upper panel, the cosine functions as results of the inversion schemes are plotted: the mean result of the Newton algorithm in dashed line, the REGINN result in dashed line with dots and the overall mean in a straight line. In the lower panels the measured temperature (left) and thermal diffusivity (right) are depicted. The resulting temperature-depth profiles from the modeling with the inversion results (the line styles are the same as above) are plotted together with the measured temperatures in the bottom left panel.

diffusivity. Before the obtained reconstruction results are discussed, the desired accuracy in geophysical usage needs to be determined.

\subsection{Desired accuracy of the reconstructed parameters}

In comparison to the measured water temperatures (see MARNET, 2014) it is obvious that the mathematical model, Eq. (6), neglects all periods smaller than 1 year.

The average temperature $A$ varied about $0.5 \mathrm{~K}$ between the years 2010 and 2011 and the amplitude about $0.8 \mathrm{~K}$ at the station FINO1 in the German North Sea. Similar results can be obtained for other stations in this region. Thus, an accuracy better than $1 \mathrm{~K}$ for the parameters $A$ and $B$ in the reconstruction is sufficient for the German North Sea.

However, for the usage of the presented method in other areas (like the Arctic Ocean) the accuracy level needs to be based on the relative error - at least for the two temperaturerelated parameters: reconstruction of a parameter of the order of $0.1 \mathrm{~K}$ with an accuracy of $\pm 1 \mathrm{~K}$ is not useful. In the experiments with artificial data sets, a relative error of magnitude around or slightly less than the relative data error was achieved. The above stated differences in the recorded bottom water temperature at the station FINO1 yield a relative change of about $5 \%$ in the average temperature and $10 \%$ in the amplitude.
The day of the annual minimum only changed about 1 day at FINO1, but was most difficult to reconstruct in all the experiments seen in Sect. 5. This is clearly due to the fact that the cosine function has a small first derivative around the extrema. Thus, the function Eq. (6) does not change a lot in the weeks around the annual minimum, e.g., it remains over 3 weeks in the lowest $1 \%$ of the covered temperature interval.

Considering all this, the reconstruction of the parameters should be better than $A \pm 5 \%, B \pm 10 \%$ and $d \pm 10$ days.

\subsection{Achieved accuracy of the reconstructed parameters}

For the real data sets, a noise level of $1 \%$ was assumed. While different noise levels were considered in the experiments with artificial data, only the obtained information on data with $1 \%$ noise is relevant for the applicability on real data. As seen in Table 1, SDs of $A \pm 0.2 \mathrm{~K}, B \pm 0.5 \mathrm{~K}$ and $d \pm 2.5$ were obtained for both algorithms. In relative errors this equals $A \pm 2$ and $B \pm 8 \%$. Thus, the desired accuracy was achieved with only 20 repeated inversions. By taking the overall mean of all reconstructions from both algorithms, the reconstruction error could be further decreased. Here, the variances and SDs increased, but the obtained results were closer to the exact parameter values.

As the function of the bottom water temperature was expanded to a cutoff Fourier series, the variances increased to $A \pm 0.5 \mathrm{~K}, B \pm 1.5 \mathrm{~K}$ and $d \pm 4.8$ days (see Table 1 ) or, in 
relative errors, $A \pm 6$ and $B \pm 24 \%$. Here, the amplitude was less accurate than desired. Still, the mean of the reconstructed values was accurate within the desired interval.

As the variances were smaller for less noisy data in both experiments, it can be concluded that both algorithms yield stable results for data with a noise level of $\leq 1 \%$ and for a bottom water temperature function which varies from a plain cosine by up to $8 \%$ (see Table 1). For higher noise levels in the data or the forcing function, the methods still converged but were not accurate enough.

\subsection{Applicability to real data}

The experiments with artificial data suggested a stable method whose accuracy could be increased by executing the algorithms repeatedly and using a mean value of results from both algorithms. The reconstruction error did not increase too much, when the function for the bottom water temperature was changed to a cutoff Fourier series; the main parameters were still reconstructed sufficiently accurately, i.e., the relative errors were smaller than $5 \%$ for the mean temperature and $10 \%$ for the amplitude and the day of the annual minimum was reconstructed within 10 days.

Using real data sets, the general form of the bottom water temperature deviation in the area of interest needs to be studied carefully. As mentioned above some areas in the Baltic Sea cannot be modeled with our simple model. The data sets from the North Sea, as the one introduced in Sect. 5.3 yielded stable results, but with rather large variances. However, the results match with the recorded water temperatures. The differences of the results from the two inversion algorithms to the graphically obtained parameter values (used from Müller et al., 2013) are within the desired error bounds. Here, the greatest differences occurred in the day of the annual minimum but, as already discussed in Sect. 6.1, it is not possible to determine it more precisely while $A$ and $B$ are only accurate to $1 \mathrm{~K}$.

Lastly, the results from the inversion of the data set west of Greenland have the smallest variances, proposing reliable values. Other surveys on the temperature (and salinity) of Baffin Bay and Davis Strait gave similar temperature values (see e.g., Ribergaard, 2008). As there are currently no long-term measurements, the results of this method are of scientific value, if one trusts them. It is important to note that matching the recorded temperatures is not the same as being exact: the recorded temperatures are also measurements with errors and they were not recorded at exactly the same locations as where the data sets were taken.

Before reconstructing the bottom water temperatures from real data sets, one should carefully consider if the simple model, Eq. (6), is applicable for said area. If it is already known that the sea is layered and not well mixed or if there are strong currents along the sea floor, this method will not give reasonable results. If one finds unreasonable results, this should be interpreted as a strong hint that the simple model and hence the method are not applicable. The algorithms differ in their approach to solve the linearized system and therefore obtain different results if the data are not exact or the function for the bottom water temperature is not of the simple form as Eq. (6). These differences in the results of the algorithms are useful to gain an insight into the uncertainty of the results that is linked to measurement noise and modeling errors. Thus, the method uses the overall mean values from both algorithms and the variances can be used as an indicator for the applicability of the simple model for the bottom water temperature function.

\subsection{Future work}

A major point with the reconstruction of real data sets was that the simple model for the bottom water temperature does not fit to all areas. Hence, a main topic in further research will be the generalization of the bottom water temperature model. The implementation of the inversion algorithms can be easily adapted to reconstruct the parameter vector of other periodic functions. The Fourier series, introduced in Sect. 5.2, was a reasonable start. The more coefficients of the Fourier series to be reconstructed, the more sensors are needed to get a full-rank derivative. For regions with more noise in the bottom water temperature deviation, such as the Baltic Sea, the smaller periods could possibly generate more realistic data and thus improve the reconstruction results for such data sets.

A piece-wise constant function as in the large-scale climate history reconstruction may also be used. Such a model is then possibly capable of reconstructing aperiodic events in the most recent water temperature changes. This will be of great interest for the Baltic Sea (e.g., to identify inflows from the North Sea over the Danish Straits) or the Arctic Sea (e.g., to indicate cold water discharge due to iceberg calving events). Simultaneous inversion of the background heat flow and the bottom water temperature should also be considered. The iterative Newton algorithm is possibly not suitable for these higher dimensional problems, but other algorithms and approaches from climate history reconstruction can be built upon (Shen and Beck, 1991; Chouinard et al., 2007).

\section{Conclusions}

The presented method yields stable results for artificial data with an accuracy within the bounds of the artificial noise level. The experiments with real data sets are very promising. The SDs are small for all data sets and the results matched the measured bottom water temperature variations from the monitoring stations (where available).

These results may be of major interest for oceanographers because they can provide oceanographic information for regions where long-term monitoring is not possible or too expensive. However, before applying the method to other re- 
gions, the validity of the simple model for the bottom water temperature needs to be carefully discussed.

Acknowledgements. This work would not have been possible without the kind permission of NSW (Norddeutsche Seekabelwerke $\mathrm{GmbH}$ ) and GEUS (De Nationale Geologiske Undersøgelser for Danmark og Grønland) to use the data sets measured on cruises with partnership of FIELAX.

The authors thank M. A. Morales Maqueda and an anonymous reviewer for their very helpful comments.

The article processing charges for this open-access publication were covered by the University of Bremen.

Edited by: J. M. Huthnance

\section{References}

Beardsmore, G. R. and Cull, J. P.: Crustal Heat Flow: a Guide to Measurement and Modelling, Cambridge University Press, New York, 336 pp., 2001.

Brakelmann, H. and Stammen, J.: Thermal Analysis of Submarine Cable Routes: LSM or FEM?, IEEE-conference PECon, Putra Jaya, Malaysia, 560-565, 2006.

Bullard, E. C.: Heat Flow in South Africa, Proc. R. Soc. Lond. A, 173, 474-502, 1939.

Bundesamt für Seeschifffahrt und Hydrographie: MARNETMessnetz, available at: http://www.bsh.de/de/Meeresdaten/ Beobachtungen/MARNET-Messnetz/index.jsp (last access: 6 June 2014), 2014.

Chouinard, C., Fortier, R., and Mareschal, J.-C.: Recent climate variations in the Subarctic inferred from three borehole temperature profiles in Northern Quebec, Canada, Earth Planet. Sc. Lett., 263, 355-369, 2007.

Clauser, C.: Geothermal energy, in: Landolt-Börnstein, Group VIII: Advanced Materials and Technologies, vol. 1: Energy Technologies, Subvol. C: Renewable Energies, edited by: Heinloth, K., Springer Verlag, Heidelberg-Berlin, 493-604, 2006.

Davis, E. E., Wang, K., Becker, K., and Yashayaev, I.: Deepocean temperature variations and implications for errors in sea floor heat flow determinations, J. Geophys. Res., 108, 2034, doi:10.1029/2001JB001695, 2003.

Dillon, M., Müller, C., and Usbeck, R.: Acquiring thermal conductivity data from shear-resistant sediments, Sea Technol., 53, 5761, 2012.

Evans, L. C.: Partial Differential Equations, American Mathematical Society, Providence, 663 pp., 2010.
Hamamoto, H., Yamano, M., and Goto, S.: Heat flow measurement in shallow seas through long-term temperature monitoring, Geophys. Res. Lett., 32, L21311, doi:10.1029/2005GL024138, 2005.

Hanke-Bourgeois, M.: Grundlagen der Numerischen Mathematik und des Wissenschaftlichen Rechnens, Vieweg+Teubner, Wiesbaden, 840 pp., 2009.

Hartmann, A. and Villinger, H.: Inversion of marine heat flow measurements by expansion of the temperature decay function, Geophys. J. Int., 148, 628-636, 2002.

Hyndman, R. D., Davis, E. E., and Wright, J. A.: The measurement of marine geothermal heat flow by a multipenetration probe with digital acoustic telemetry and insitu thermal conductivity, Mar. Geophys. Res., 4, 181-205, 1979.

Jaupart, C. and Mareschal, J.-C.: Heat Generation and Transport in the Earth, Cambridge University Press, New York, 464 pp., 2011.

Lowrie, W.: Fundamentals of Geophysics, Cambridge University Press, New York, 381 pp., 2007.

Müller, C, Miesner, F., Usbeck, R., and Schmitz, T.: 2 K-criterion: measuring and modelling temperatures and thermal conductivities/diffusivities in shallow marine sediments, Conference on Maritime Energy 2013, TUHH, Hamburg, 475-490, 2013.

Omstedt, A. and Axel, L. B.: Modelling the seasonal, interannual, and long-term variations of salinity and temperature in the Baltic proper, Tellus A, 50, 637-652, 1998.

Rhode, J., Tett, P., and Wulff, F.: The Baltic and North Seas: a regional review of some important physical-chemical-biological interaction processes, in: The Seas, vol. 14, chap. 26, edited by: Robinson, A. R. and Brink, K. H., Harvard University Press, 1029-1071, 2004.

Ribergaard, M. H.: Oceanographic Investigations off West Greenland 2011, Danish Meteorological Institute Centre for Ocean and Ice, Copenhagen, 2011.

Rieder, A.: On the regularization of nonlinear ill-posed problems via inexact Newton iterations, Inverse Probl., 15, 309-327, 1999a.

Rieder, A.: On convergence rates of inexact Newton regularizations, Numer. Math., 88, 347-365, 1999b.

Rieder, A.: Keine Probleme mit Inversen Problemen: Eine Einführung in stabile Lösungen, Vieweg+Teubner Verlag, Wiesbaden, 2003.

Shen, P. Y. and Beck, A. E.: Least Squares Inversion of Borehole Temperature Measurements in Functional Space, J. Geophys. Res., 96, 19965-19979, 1991.

The International Heat Flow Commission: Global Heat Flow Database, available at: http://www.heatflow.und.edu/index2.html (last access: 23 July 2014), 2014.

Wang, K. and Beck, A. E.: Heat flow measurement in lacrustine or oceanic sediments without recording bottom temperature variations, Geophys. Res., 92, 12837-12845, 1987. 